\title{
Annihilator of Generalized Derivations with Power Values in Rings and Algebras
}

\author{
Md Hamidur Rahaman*
}

\begin{abstract}
Let $\mathcal{F}, \mathcal{G}$ be two generalized derivations of prime ring $\mathcal{R}$ with characteristic different from 2 with associated derivations $\mathcal{D}_{1}$ and $\mathcal{D}_{2}$ respectively. We use the symbols $\mathcal{C}=\mathcal{Z}(\mathcal{U})$ and $\mathcal{U}$ to denote the the extended centroid of $\mathcal{R}$ and Utumi ring of quotient of $\mathcal{R}$ respectively. Let $0 \neq a \in \mathcal{R}$ and $\mathcal{F}$ and $\mathcal{G}$ satisfy $a\left\{(\mathcal{F}(x y)+\mathcal{G}(y x))^{m}-[x, y]^{n}\right\}=0$ for all $x, y \in \mathcal{J}$, a nonzero ideal, where $m$ and $n$ are natural numbers. Then either $\mathcal{R}$ is commutative or there exists $c, b \in \mathcal{U}$ such that $\mathcal{F}(x)=c x$ and $\mathcal{G}(x)=b x$ for all $x \in \mathcal{R}$.

Keywords: Semiprime rings; Generalized derivations; extended centroid; Utumi quotient ring.

AMS Subject Classification (2020): Primary: 16W25 ; Secondary: 16N60; 15A27.

${ }^{*}$ Corresponding author
\end{abstract}

\section{Introduction}

For any $x, y \in \mathcal{R}$ we use the symbol $[x, y]$ to denote the commutator $x y-y x$ and $x \circ y$ to denote ant-icommutator $x y+y x$. Recall that a ring $\mathcal{R}$ is prime if for any $a, b \in R, a \mathcal{R} b=\{0\}$ implies that either $a=0$ or $b=0$ and is semiprime if for any $a \in \mathcal{R}, a \mathcal{R} a=\{0\}$ implies that $a=0$. A map $\mathcal{D}: \mathcal{R} \rightarrow \mathcal{R}$ is said to be a derivation if $\mathcal{D}$ is additive and $\mathcal{D}(x y)=\mathcal{D}(x) y+x \mathcal{D}(y)$ for all $x, y \in \mathcal{R}$. If $\mathcal{D}$ can be written as $\mathcal{D}(x)=[a, x]$ for all $x \in \mathcal{R}$, then $\mathcal{D}$ is called an inner derivation for some $a \in \mathcal{R}$. Brešar [2] brought out the definition of generalized derivation. A map $\mathcal{F}: \mathcal{R} \rightarrow \mathcal{R}$ is said to be a generalized derivation if it is additive and satisfies $\mathcal{F}(x y)=\mathcal{F}(x) y+x \mathcal{D}(y)$ for all $x, y \in \mathcal{R}$ and a derivation $\mathcal{D}$.

Daif et al. [4, Theorem 2] showed that if $\mathcal{R}$ is a semiprime ring with $\{0\} \neq \mathcal{J}$ ideal and $\mathcal{D}$ is a derivation of $\mathcal{R}$ such that $\mathcal{D}([x, y])=[x, y]$ for any $x, y \in \mathcal{J}$, then $\mathcal{J}$ is contained in the centre of $\mathcal{R}$. Later Quadri et al. [12] discussed the commutativity of prime rings for generalized derivation instead of derivation. Further, Dhara [7] studied the result of Quadri et al. in semiprime ring. Filippis et al. [6] studied that if $\mathcal{F}$ satisfies $(\mathcal{F}([x, y]))^{n}=[x, y]$ for all $x, y \in \mathcal{J}$, where $\{0\} \neq \mathcal{J}$ is an ideal of a prime ring $\mathcal{R}, n$ is a fixed natural number and $\mathcal{F}$ is a generalized derivations of $\mathcal{R}$, they conclude that either $\mathcal{D}=0, n=1$ and $\mathcal{F}(x)=x R$ for all $x \in \mathcal{R}$ or $\mathcal{R}$ is commutative.

As $\mathcal{F}$ is additive, further the above identity can be written as $(\mathcal{F}(x y)-\mathcal{F}(y x))^{n}=[x, y]$. Form this point of view there is a question what happen if we take two generalized derivations instead of generalized derivation. Following this line, we prove:

Theorem 1.1. Let $\mathcal{R}$ be a prime ring with characteristic different from $2, \mathcal{F}$ and $\mathcal{G}$ are generalized derivations of $\mathcal{R}$. Let $0 \neq a \in \mathcal{R}$ and $\mathcal{F}$ and $\mathcal{G}$ satisfy $a\left\{(\mathcal{F}(x y)+\mathcal{G}(y x))^{m}-[x, y]^{n}\right\}=0$ for all $x, y \in \mathcal{J}$, a nonzero ideal, where $m$ and $n$ are natural numbers. Then we have exactly one of the following:

1. $\mathcal{R}$ is commutative;

2. there exists $c, b \in \mathcal{U}$, Utumi ring of quotient of $\mathcal{R}$ such that $\mathcal{F}(x)=c x$ and $\mathcal{G}(x)=$ bx for all $x \in \mathcal{R}$.

Received : 09-10-2019, Accepted : 23-05-2020 
Theorem 1.2. Let $\mathcal{R}$ be a 2-torsion free semiprime ring, $\mathcal{F}$ and $\mathcal{G}$ are generalized derivations of $\mathcal{R}$ with associated derivations $\mathcal{D}_{1}, \mathcal{D}_{2}$ respectively. Let $0 \neq a \in \mathcal{R}$ and $\mathcal{F}, \mathcal{G}$ satisfy $a\left\{(\mathcal{F}(x y)+\mathcal{G}(y x))^{m}-[x, y]^{n}\right\}=0$ for all $x, y \in \mathcal{J}$, a nonzero ideal, where $m$ and $n$ are natural numbers. Then $\mathcal{R}$ contains an ideal which is central.

\section{The results on two sided ideals}

Theorem 1.1 Let $\mathcal{R}$ be a prime ring with characteristic different from $2, \mathcal{F}$ and $\mathcal{G}$ are generalized derivations of $\mathcal{R}$. Let $0 \neq a \in \mathcal{R}$ and $\mathcal{F}$ and $\mathcal{G}$ satisfy $a\left\{(\mathcal{F}(x y)+\mathcal{G}(y x))^{m}-[x, y]^{n}\right\}=0$ for all $x, y \in \mathcal{J}$, a nonzero ideal, where $m$ and $n$ are natural numbers. Then we have exactly one of the following:

1. $\mathcal{R}$ is commutative;

2. there exists $c, b \in \mathcal{U}$, Utumi ring of quotient of $\mathcal{R}$ such that $\mathcal{F}(x)=c x$ and $\mathcal{G}(x)=b x$ for all $x \in \mathcal{R}$.

Proof If both $\mathcal{F}$ and $\mathcal{G}$ are zero, then $a[x, y]^{n}=0$ for all $x, y \in \mathcal{J}$. Since $[x, y]$ is multilinear, by [16], we have $a[x, y]=0$. Replacing $x$ by $z x$ to get $a z[x, y]=0$. Using primeness of $\mathcal{R}$ and $a \neq 0$, we have $[x, y]=0$ and hence $\mathcal{R}$ is commutative. Suppose atleast one of $\mathcal{F}, \mathcal{G} \neq 0$, then from the hypothesis, we have

$$
a\left\{(\mathcal{F}(x y)+\mathcal{G}(y x))^{m}-[x, y]^{n}\right\}=0 \text { for all } x, y \in \mathcal{J}
$$

By [10], $\mathcal{F}(x)=c x+\mathcal{D}_{1}(x)$ and $\mathcal{G}(x)=b x+\mathcal{D}_{2}(x)$ for some $c, b \in \mathcal{U}$, for all $x \in \mathcal{U}$ and derivations $\mathcal{D}_{1}$ and $\mathcal{D}_{2}$. Hence $\mathcal{J}$ satisfies

$$
a\left\{(\mathcal{F}(x y)+\mathcal{G}(y x))^{m}-[x, y]^{n}\right\}=0
$$

By [3, Theorem 2], $\mathcal{U}$ satisfies this GPI, we have

$$
\left.a\left\{\left(c x y+D_{1}(x y)\right)+\left(b y x+D_{2}(y x)\right)\right)^{m}-[x, y]^{n}\right\}=0 \text { for all } x, y \in \mathcal{U} .
$$

Now we have the following cases:

Case I: Let $\mathcal{D}_{1}$ and $\mathcal{D}_{2}$ are inner derivations of $\mathcal{U}$, i.e $\mathcal{D}_{1}(x)=[p, x]$ and $\mathcal{D}_{2}(x)=[q, x]$ for all $x \in \mathcal{U}$ and for some $p, q \in \mathcal{U}$. Then our identity $\left.a\left\{\left(c x y+D_{1}(x y)\right)+\left(b y x+D_{2}(y x)\right)\right)^{m}-[x, y]^{n}\right\}=0$ becomes

$$
\left.a\{(c x y+[p, x y])+(b y x+[q, y x]))^{m}-[x, y]^{n}\right\}=0 \text { for all } x, y \in \mathcal{U} .
$$

Hence $\left.a\{(a x y+[p, x y])+(b y x+[q, y x]))^{m}-[x, y]^{n}\right\}=0$ is a nontrivial generalized polynomial identity (GPI) for $\mathcal{U}$. Denote by $\mathcal{H}$ either the algebraic closure of $\mathcal{C}$ or $\mathcal{C}$ according as $\mathcal{C}$ is either infinite or finite respectively. By $([9$, Proposition]), $\left.a\{(c x y+[p, x y])+(b y x+[q, y x]))^{m}-[x, y]^{n}\right\}=0$ is also a GPI for $\mathcal{U} \otimes_{\mathcal{C}} \mathcal{H}$. By [17, Theorem 2.5 and Theorem 3.5], $\mathcal{U} \otimes_{\mathcal{C}} \mathcal{H}$ is centrally closed prime $\mathcal{H}$-algebra, by replacing $\mathcal{R}, \mathcal{C}$ with $\mathcal{U} \otimes_{\mathcal{C}} \mathcal{H}$ and $\mathcal{H}$, respectively, we may assume that $\mathcal{R}$ is centrally closed and $\mathcal{C}$ is either finite or algebraically closed. By Martindale's theorem [18], $\mathcal{R}$ is then a primitive ring with nonzero socle $\mathcal{E}$ with $\mathcal{C}$ as the associated division ring. Hence by Jacobson's theorem $\left[19\right.$, p.75] $\mathcal{R} \cong \mathcal{M}_{k}(\mathcal{C})$. If $k=1$, then $\mathcal{R}$ is commutative. Now assume $\operatorname{dim}_{\mathcal{C}} \mathcal{V} \geq 2$.

Now we prove that for any $u \in \mathcal{V}, u$ and $q u$ are linearly $\mathcal{C}$-dependent. Let on contrary that $u$ and $q u$ are linearly independent for some $u \in \mathcal{V}$.

If $p u$ is not a member of the span of $\{u, q u\}$, then $\{u, p u, q u\}$ is independent. By the density of ring $\mathbb{R}$, there exist $y, x \in \mathbb{R}$ such that

$$
x q u=-u, x u=0, y p u=u, y u=u, x p u=0, y q u=u .
$$

Then multiplying (2.4) by $u$ from rihgt to have

$$
\left.0=a\{(c x y+[p, x y])-(b y x+[q, y x]))^{m}-[x, y]^{n}\right\} u=0=a u .
$$

If for any $v \in \mathcal{V},\{u, v\}$ is linearly $\mathcal{C}$-dependent, then $a v=0$. Since $a \neq 0$, there exists $w \in \mathcal{V}$ such that $a w \neq 0$ and so $\{w, v\}$ are linearly $\mathcal{C}$-independent. Also $a(w+v)=a w \neq 0$ and $a(w-v)=a w \neq 0$. By the above argument, it follows that $w$ and $c w$ are linearly $\mathcal{C}$-dependent, as are $\{w+v, c(w+v)\}$ and $\{w-v, c(w-v)\}$. Therefore there exist $\gamma_{w}, \gamma_{w+v}, \gamma_{w-v} \in \mathcal{C}$ such that

$$
q w=\gamma_{w} w, \quad(w+v)=\gamma_{w+v}(w+v), \quad q(w-v)=\gamma_{w-v}(w-v)
$$

Thus we have

$$
\gamma_{w} w+q v=\gamma_{w+v} w+\gamma_{w+v} v
$$


and

$$
\gamma_{w} w-q v=\gamma_{w-v} w-\gamma_{w-v} v
$$

Now (2.5) and (2.6) together yields

$$
\left(2 \gamma_{w}-\gamma_{w+v}-\gamma_{w-v}\right) w+\left(\gamma_{w-v}-\gamma_{w+v}\right) v=0
$$

and

$$
2 q v=\left(\gamma_{w+v}-\gamma_{w-v}\right) w+\left(\gamma_{w+v}+\gamma_{w-v}\right) v .
$$

By (2.7), and since $\{w, v\}$ are $\mathcal{C}$-independent, $2 \gamma_{w}-\gamma_{w+v}-\gamma_{w-v}=0$ and $\gamma_{w-v}-\gamma_{w+v}=0$. These relations imply by using char $(\mathcal{R}) \neq 2$, that $\gamma_{w}=\gamma_{w+v}=\gamma_{w-v}$. By (2.8) it follows $q v=\gamma_{w} v$. This leads to a contradiction with the fact that $\{v, q v\}$ is linear $\mathcal{C}$-independent.

In light of this, we may assume that for any $v \in \mathcal{V}$ there exists a suitable $\gamma_{v} \in \mathcal{V}$ such that $q v=\gamma_{v} v$, and standard argument shows that there is $\gamma \in \mathcal{C}$ such that $q v=\gamma v$ for all $v \in \mathcal{V}$. Then by standard argument, we have $q \in \mathcal{C}$. similarly with neccessary variation, we can show that $p \in \mathcal{C}$.

Case 2 Let $\mathcal{D}_{1}$ and $\mathcal{D}_{2}$ are not both inner derivations of $\mathcal{U}$. Then $\mathcal{D}_{2}(y)=[p, y]+\beta \mathcal{D}_{1}(y)$ for some $p \in \mathcal{U}$ and $\beta \in \mathcal{C}$. If either $\beta=0$ or $\mathcal{D}_{2}$ is inner, then $\mathcal{D}_{1}$ is also inner which contradicts. So, $\beta \neq 0$ as wel as $\mathcal{D}_{2}$ is not inner. Then by (2.3), we have

$$
a\left\{\left(c x y+\mathcal{D}_{1}(x) y+x \mathcal{D}_{1}(y)\right)+\left(b y x+[p, y x]+\beta\left(\mathcal{D}_{1}(y) x+y \mathcal{D}_{1}(x)\right)\right)^{m}-[x, y]^{n}\right\}=0
$$

for any $y, x \in \mathcal{U}$.

By the use of Kharchenko's Theorem [8], we have either $\mathcal{D}_{1}$ is inner or $\mathcal{U}$ satisfies $a\left\{\left(c x y+x_{1} y+x y_{1}\right)+(b y x+\right.$ $\left.\left.\left.[p, y x]+\beta\left(y_{1} x+y x_{1}\right)\right)\right)^{m}-[x, y]^{n}\right\}$ i.e

$$
\left.a\left\{\left(c x y+x_{1} y+x y_{1}\right)+\left(b y x+[p, y x]+\beta\left(y_{1} x+y x_{1}\right)\right)\right)^{m}-[x, y]^{n}\right\}=0
$$

for any $y, x, y_{1}, x_{1} \in \mathcal{U}$.

If $\mathcal{D}_{1}$ is inner then $\mathcal{D}_{2}$ will be a inner derivation of form $\mathcal{D}_{2}(y)=[p+\beta q, y]$ for some $p, q \in \mathcal{U}$ which is a contradiction. In particular, putting $y=0$ in (2.9), we have

$$
a\left(x y_{1}+\beta y_{1} x\right)^{m}=0 \text { for any } x, y_{1} \in \mathcal{J} .
$$

Since $x y_{1}+\beta y_{1} x$ is multilinear polynomial, by [16], we have $a\left(x y_{1}+\beta y_{1} x\right)=0$. Further, this can be written as $a\left(x y_{1}-y_{1} x+\beta y_{1} x+y_{1} x\right)=0=a\left(\left(x y_{1}-y_{1} x\right)+\left(y_{1} x+\beta y_{1} x\right)\right)=a\left(x y_{1}-y_{1} x\right)$. By primeness of $\mathcal{R}$, we have $[\mathcal{R}, \mathcal{R}]=\{0\}$ and hence $\mathcal{R}$ is commutative.

Case 3 Now assume both $\mathcal{D}_{1}$ and $\mathcal{D}_{2}$ are Outer. By Kharchenko's Theorem [8], we have

$$
\left.a\left\{\left(c x y+x_{1} y+x y_{1}\right)+\left(b y x+y_{2} x+y x_{2}\right)\right)^{m}-[x, y]^{n}\right\}=0
$$

for any $y_{1}, x_{1}, y, x, y_{2}, x_{2} \in \mathbb{U}$. For $y=0$, we have

$$
a\left(x y_{1}+y_{2} x\right)^{m}=0
$$

Since $x y_{1}+y_{2} x$ is multilinear polynomial, by [16], we have $a\left(x y_{1}+y_{2} x\right)=0$. By primeness of $\mathcal{R}$, we have $\mathcal{R} \circ \mathcal{R}=\{0\}$ and hence $\mathcal{R}$ is commutative.

Now we have the following corollaries

Corollary 2.1. Let $\mathcal{F}$ and $\mathcal{G}$ be two generalized derivations of $\mathcal{R}$, a prime ring having characteristic different from 2, associated with nonzero derivations and $\{0\} \neq \mathcal{J}$ an ideal of $\mathcal{R}$. Let $0 \neq a \in \mathcal{R}$ and $\mathcal{F}$ and $\mathcal{G}$ satisfy $a\left\{(\mathcal{F}(x y)+\mathcal{G}(y x))^{m}-[x, y]^{n}\right\}=0$ for all $x, y \in \mathcal{J}$ and for some fixed natural numbers $m, n$. Then $\mathcal{R}$ is commutative.

Corollary 2.2. Let $\mathcal{D}_{1}$ and $\mathcal{D}_{2}$ be two derivations of $\mathcal{R}$, a prime ring having characteristic different from 2 and $\{0\} \neq \mathcal{J}$ an ideal of $\mathcal{R}$. Let $0 \neq a \in \mathcal{R}$ and $\mathcal{D}_{1}$ and $\mathcal{D}_{2}$ satisfy $a\left\{\left(\mathcal{D}_{1}(x y)+\mathcal{D}_{2}(y x)\right)^{m}-[x, y]^{n}\right\}=0$ for all $x, y \in \mathcal{J}$ and for some fixed natural numbers $m, n$. Then $\mathcal{R}$ is commutative.

In the following example, we demonstrate that primeness of the ring is essential in the hypothesis of the Theorem1.1. 
Example 2.1. Let $\mathcal{R}_{1}$ be any commutative ring. Let $\mathcal{R}=\left\{\left(\begin{array}{cc}a & b \\ 0 & c\end{array}\right) \mid a, b, c \in \mathcal{R}_{1}\right\}$ and $\mathcal{J}=\left\{\left(\begin{array}{ll}0 & a \\ 0 & 0\end{array}\right) \mid\right.$ $\left.a \in \mathcal{R}_{1}\right\}$. Define the following maps: $\mathcal{F}\left(\begin{array}{ll}a & b \\ 0 & c\end{array}\right)=\left(\begin{array}{cc}a & c \\ 0 & 0\end{array}\right), \mathcal{G}\left(\begin{array}{cc}a & b \\ 0 & c\end{array}\right)=\left(\begin{array}{cc}a & a+b \\ 0 & 0\end{array}\right), \mathcal{D}_{1}\left(\begin{array}{cc}a & b \\ 0 & c\end{array}\right)=$ $\left(\begin{array}{cc}0 & -b \\ 0 & 0\end{array}\right)$ and $\mathcal{D}_{2}\left(\begin{array}{ll}a & b \\ 0 & c\end{array}\right)=\left(\begin{array}{cc}0 & a-c \\ 0 & 0\end{array}\right)$. Then $\mathcal{F}$ and $\mathcal{G}$ are generalized derivations with associated derivations $\mathcal{D}_{1}$ and $\mathcal{D}_{2}$ respectively satisfying $a\left\{(\mathcal{F}(x y)+\mathcal{G}(y x))^{m}-[x, y]^{n}\right\}=0$ for all $x, y \in \mathcal{J}$, where $m \geq 1, n \geq 1$ are fixed integer. Then neither $\mathcal{R}$ is commutative nor $\mathcal{F}$ and $\mathcal{G}$ can be written as $\mathcal{F}(x)=a x$ and $\mathcal{G}(x)=b x$ for all $x \in \mathcal{R}$ because of $\mathcal{D}_{1}$ and $\mathcal{D}_{2}$ are nonzero.

\section{Results on semiprime rings}

Theorem 1.2 Let $\mathcal{R}$ be a 2-torsion free semiprime ring, $\mathcal{F}$ and $\mathcal{G}$ are generalized derivations of $\mathcal{R}$ with associated derivations $\mathcal{D}_{1}, \mathcal{D}_{2}$ respectively. Let $0 \neq a \in \mathcal{R}$ and $\mathcal{F}$ and $\mathcal{G}$ satisfy $a\left\{(\mathcal{F}(x y)+\mathcal{G}(y x))^{m}-[x, y]^{n}\right\}=0$ for all $x, y \in \mathcal{J}$, a nonzero ideal, where $m$ and $n$ are fixed natural numbers. Then $\mathcal{R}$ contains an ideal which is central.

Proof By [3] and [9], $\mathcal{J}, \mathcal{R}$ and $\mathcal{U}$ satisfy the same GPIs as well as same differential identities. By [10], $\mathcal{F}(x)=$ $c x+\mathcal{D}_{1}(x)$ and $\mathcal{G}(x)=b x+\mathcal{D}_{2}(x)$ for some $c, b \in \mathcal{U}$ and derivations $\mathcal{D}_{1}$ and $\mathcal{D}_{2}$. Hence

$$
a\left\{\left(\left(c x y+\mathcal{D}_{1}(x y)\right)+\left(b y x+\mathcal{D}_{2}(y x)\right)^{m}-[x, y]^{n}\right\}=0 \text { for all } x, y \in \mathcal{U} .\right.
$$

Let $\mathcal{P}(\mathcal{C})$ denotes a collection of all maximal ideals of $\mathcal{C}$ and $\mathcal{M} \in \mathcal{P}(\mathcal{C})$. By the theory of orthogonal completions for semiprime rings $([9$, p.31-32]), $\mathcal{M U}$ is a prime ideal of $\mathcal{U}$ which is invariant under all derivations of $\mathcal{U}$. By $[1$, Lemma 1 and Theorem 1], we have $\bigcap\{\mathcal{M U} \mid \mathcal{M} \in \mathcal{M}(\mathcal{C})\}=0$. Set $\overline{\mathcal{U}}=\mathcal{U} / \mathcal{M U}$. Then $\mathcal{D}_{1}$ and $\mathcal{D}_{2}$ induce the following derivations $\overline{\mathcal{D}_{1}}$ and $\overline{\mathcal{D}_{2}}$ on $\overline{\mathcal{U}}$ whic is defined as $\overline{\mathcal{D}_{1}}(\bar{x})=\overline{\mathcal{D}_{1}(x)}$ and $\overline{\mathcal{D}_{2}}(\bar{x})=\overline{\mathcal{D}_{2}(x)}$ for all $x \in \overline{\mathcal{U}}$. Therefore,

$$
\bar{a}\left\{\left(\left(\bar{c} \bar{x} \bar{y}+\overline{\mathcal{D}}_{1}(\bar{x} \bar{y})\right)+\left(\bar{b} \bar{y} \bar{x}+\overline{\mathcal{D}}_{2}(\bar{y} \bar{x})\right)\right)^{m}-[\bar{x}, \bar{y}]^{n}\right\}=0
$$

for all $\bar{x}, \bar{y} \in \overline{\mathcal{U}}$. Using Theorem 1.1, we have simultaneously either $[\mathcal{U}, \mathcal{U}] \subseteq \mathcal{M U}$ or $\mathcal{D}_{1}(\mathcal{U}) \subseteq \mathcal{M U}$ as well as either $[\mathcal{U}, \mathcal{U}] \subseteq \mathcal{M U}$ or $\mathcal{D}_{2}(\mathcal{U}) \subseteq \mathcal{M U}$. This gives that $\mathcal{D}_{1}(\mathcal{U})[\mathcal{U}, \mathcal{U}] \subseteq \mathcal{M U}$ for all $\mathcal{M} \in \mathcal{P}(\mathcal{C})$ as well as $\mathcal{D}_{2}(\mathcal{U})[\mathcal{U}, \mathcal{U}] \subseteq \mathcal{M U}$ for all $\mathcal{M} \in \mathcal{P}(\mathcal{C})$. In either case we have $\mathcal{D}_{i}(\mathcal{U})[\mathcal{U}, \mathcal{U}] \subseteq \mathcal{M U}$ for all $\mathcal{M} \in \mathcal{P}(\mathcal{C}), i=1,2$ and hence $\mathcal{D}_{i}(\mathcal{U})[\mathcal{U}, \mathcal{U}]=0$. Particularly, $\mathcal{D}_{i}(\mathcal{R})[\mathcal{R}, \mathcal{R}]=0=\left[\mathcal{D}_{i}(\mathcal{R}), \mathcal{R}\right] \mathcal{R}\left[\mathcal{D}_{i}(\mathcal{R}), \mathcal{R}\right]=0$. As $\mathcal{R}$ is a semiprime ring, we obtain that $\left[\mathcal{D}_{i}(\mathcal{R}), \mathcal{R}\right]=$ 0 . Then by [20, Theorem 3], $\mathcal{R}$ contains a nonzero central ideal.

\section{Results on Banach algebras}

Singer et al. [14] showed that the image of a noncommutative Banach algebra under continuous derivation is contained in radical of the algebra. Sinclair [13] proved that every primitive ideals of the algebra is invariant under continuous derivation of Banach algebra. Recently, Park [11] proved that if $\mathcal{D}$ is a continuous linear derivation of a noncommutative Banach algebra $\mathcal{A}$ satisfies $[[\mathcal{D}(x), x], \mathcal{D}(x)] \in \operatorname{rad}(\mathcal{A})$ for all $x \in \mathcal{A}$, then $\mathcal{D}(\mathcal{A}) \subseteq \operatorname{rad}(\mathcal{A})$. De Filippis [5] extended the Park's result to generalized derivations.

Inspire by these results we prove:

Theorem 4.1. Let $\mathcal{A}$ be a noncommutative Banach algebra and $\mathcal{F}(x)=c x+\mathcal{D}_{1}(x)$ and $\mathcal{G}(x)=b x+\mathcal{D}_{2}(x)$ are continuous generalized derivations with associated derivations $\mathcal{D}_{1}(x)$ and $\mathcal{D}_{2}(x)$ respectively. If $\mathcal{F}$ and $\mathcal{G}$ satisfy a $\left\{(\mathcal{F}(x y)+\mathcal{G}(y x))^{m}-\right.$ $\left.\left.[x, y]^{n}\right\} \in \operatorname{rad}(\mathcal{A})\right)$ for all $x, y \in \mathcal{A}$, then $\mathcal{D}_{i}(\mathcal{A}) \subseteq \operatorname{rad}(\mathcal{A})$ for $i=1,2$.

By a Banach algebra, we mean a complex normed algebra $\mathcal{A}$ whose underlying vector space is Banach space. Here Jacobson radical of $\mathcal{A}$ is defined as the intersection of all primitive ideals of $\mathcal{A}$ and we use the notation $\operatorname{rad}(\mathcal{A})$ to denote it.

Proof of Theorem 4.1 We know that left multiplication mappings are continuous. Also $\mathcal{F}, \mathcal{G}$ are continuous by hypothesis. So $\mathcal{D}_{1}$ and $\mathcal{D}_{2}$ are continuous. By [13] it is clear that primitive ideals are invariant under continuous generalized derivations $\mathcal{F}, \mathcal{G}$. Assuming $\mathcal{A} / \mathcal{P}=\overline{\mathcal{A}}$ for any primitive ideal $\mathcal{P}$. Thus generalized derivations $\mathcal{F}_{p}, \mathcal{G}_{q}: \overline{\mathcal{A}} \rightarrow \overline{\mathcal{A}}$ is defined by $\mathcal{F}_{p}(\bar{x})=\mathcal{F}_{p}(x+P)=\mathcal{F}(x)+P=a x+\mathcal{D}_{1}(x)+P$ and $\mathcal{G}_{q}(\bar{x})=\mathcal{G}_{q}(x+P)=\mathcal{G}(x)+P=$ $b x+\mathcal{D}_{2}(x)+P$ for all $\bar{x} \in \overline{\mathcal{A}}$, where $\mathcal{A} / \mathcal{P}=\overline{\mathcal{A}}$ is a factor Banach algebra. As $\mathcal{P}$ is primitive, the factor algebra $\overline{\mathcal{A}}$ is also primitive and hence it is prime and semisimple. The hypothesis $\left.a\left\{(\mathcal{F}(x y)+\mathcal{G}(y x))^{m}-[x, y]^{n}\right\} \in \operatorname{rad}(\mathcal{A})\right)$ 
yields that $\bar{a}\left\{\left(\mathcal{F}_{p}(\bar{x} \bar{y})+\mathcal{G}_{q}(\bar{y} \bar{x})\right)^{m}-[\bar{x}, \bar{y}]^{n}\right\}=\overline{0}$ for all $\bar{x}, \bar{y} \in \bar{A}$. By Theorem 1.1, we have either $\bar{A}$ is commutative or $\overline{\mathcal{D}}_{1}=\overline{0}$ and $\mathcal{D}_{2}=\overline{0}$.

Let $\overline{\mathcal{A}}$ be commutative. By [15], $\overline{\mathcal{D}_{1}}$ and $\overline{\mathcal{D}_{2}}$ are continuous in $\overline{\mathcal{A}}$. By [14], $\overline{\mathcal{D}_{1}}=\overline{0}$ and $\overline{\mathcal{D}_{2}}=\overline{0}$ in $\overline{\mathcal{A}}$. So, in both cases, we have $\overline{\mathcal{D}}_{1}=\overline{0}$ and $\overline{\mathcal{D}}_{2}=\overline{0}$ in $\overline{\mathcal{A}}$, i.e $\mathcal{D}_{i}(A) \subseteq \mathcal{P}$ for any primitive ideal $\mathcal{P}$ of $\mathcal{A}$ and hence $\mathcal{D}_{i}(A) \subseteq \operatorname{rad}(\mathcal{A})$ for $i=1,2$.

\section{References}

[1] Beidar, K. I.: Rings of quotients of semiprime rings. Vestnik Moskov. Univ. Ser I Math. Meh. (Engl. Transl:. Moscow Univ. Math. Bull.). 33,36-42 (1978).

[2] Brešar, M.: On the distance of the composition of the two derivations to be the generalized derivations. Glasgow Math. J. 33 (1), 89-93 (1991).

[3] Chuang, C. L.: GPIs having coefficients in Utumi quotient rings. Proc. Amer. Math. Soc. 103, 723-728 (1988).

[4] Daif, M. N. and Bell, H. E: Remarks on derivations on semiprime rings. Int. J. Math. \& Math. Sci. 15 (1) , 205-206 (1992).

[5] Filippis, V. De: Generalized derivations in prime rings and noncommutative Banach algebras. Bull. Korean Math. Soc. 45, 621-629 (2008).

[6] Filippis, V. De and Huang, S.: Generalized derivations on semi prime rings. Bull. Korean Math. Soc. 48 (6), 1253-1259 (2011).

[7] Dhara, B.: Remarks on generalized derivations in prime and semiprime rings. Int. J. Math. \& Math. Sc. 2010, Article ID 646587, 6 pages.

[8] Kharchenko, V. K.: Differential identity of prime rings. Algebra and Logic. 17, 155-168 (1978).

[9] Lee, T. K.: Semiprime rings with differential identities. Bull. Inst. Math. Acad. Sinica, 20 (1) , 27-38 (1992).

[10] Lee, T. K.: Generalized derivations of left faithful rings. Comm. Algebra, 27 (8), 4057-4073 (1999).

[11] Park, K. H.: On derivations in noncommutative semiprime rings and Banach algebras. Bull. Korean Math. Soc. 42, 671-678 (2005).

[12] Quadri, M. A., Khan, M. S. and Rehman, N.: Generalized derivations and commutativity of prime rings. Indian J. Pure Appl. Math. 34 (9),1393-1396 (2003).

[13] Sinclair, A. M.: Continuous derivations on Banach algebras. Proc. Amer. Math. Soc. 20, 166-170 (1969).

[14] Singer, I. M. and Wermer, J.: Derivations on commutative normed algebras. Math. Ann. 129, 260-264 (1955).

[15] Johnson, B. E. and Sinclair, A. M.: Continuity of derivations and a problem of Kaplansky. Amer. J. Math. 90, 1067-1073 (1968).

[16] Chuang, C. L. and Lee, T. K.: Rings with annihilator conditions on multilinear polynomials. Chinese J. Math. 177-185 (1996).

[17] Erickson, T. S. and Martindale III, W. S. and Osborn, J. M.: Prime nonassociative algebras. Pacific J. Math. 60, 49-63 (1975).

[18] Martindale III, W. S.: Prime rings satisfying a generalized polynomial identity. J. Algebra. 12, 576-584 (1969).

[19] Jacobson, N.: Structure of rings. Amer. Math. Soc. Colloq. Pub., 37, Amer. Math. Soc., Providence, RI, 1964.

[20] Bell, H. E.and Martindale III, W. S.: Centralizing mappings of semiprime rings. Canad. Math. Bull. 30, 92-101 (1987). 


\section{Affiliations}

MD HAMIDUR RAHAMAN

AdDress: Aligarh Muslim University, Dept. of Mathematics, 202002, Aligarh-India.

E-MAIL: rahamanhamidmath@gmail.com

ORCID ID: 0000-0003-1822-7863 